PROCEEDINGS OF THE

AMERICAN MATHEMATICAL SOCIETY

Volume 124, Number 12, December 1996, Pages 3805-3811

S 0002-9939(96)03671-4

\title{
SOME FACTS ABOUT EISENMAN INTRINSIC MEASURES. II
}

\author{
SHULIM KALIMAN
}

(Communicated by Eric Bedford)

\begin{abstract}
We construct a measure hyperbolic manifold which does not admit a Hermitian metric whose Ricci curvature is negatively bounded. We construct a C-connected Stein manifold which is not densely sub-Euclidean or Runge (in the sense of Gromov). We find some conditions under which the Eisenman intrinsic $k$-measure of a complex manifold does not change when we delete an exclusive divisor of this manifold.
\end{abstract}

\section{INTRODUCTION}

In this short note we develop further some results which were obtained in [Ka95]. Recall that for every $k=1, \ldots, m$ each complex manifold $M$ of dimension $m$ possesses $k$-dimensional Eisenman intrinsic measure $E_{k}^{M}$ which depends only on the complex structure of $M$ (see [E70]). The Eisenman intrinsic $m$-measure is called the Kobayashi-Eisenman pseudovolume of $M$. Other intrinsic measures on $M$ were introduced by Yau [Y75]. We call them the Yau $k$-measures $Y_{k}^{M}$, and $Y_{m}^{M}$ is the Yau pseudovolume respectively. The advantage of the Yau measures is that they are invariant under bimeromorphic mappings while the Eisenman measures are invariant only under biholomorphic mappings. In [Ka95] we began to study the behavior of Eisenman measures under bimeromorphic mappings and we established the following fact. If $\sigma: \tilde{M} \rightarrow M$ is the blowing-up of $M$ with centrum at a submanifold $A$ of codimension $\ell \geq 2$, then under some additional condition on $A$ the measure $E_{k}^{\tilde{M}-\tilde{A}}$ coincides with the restriction of $E_{k}^{\tilde{M}}$ to $\tilde{M}-\tilde{A}$, where $\tilde{A}$ is the exclusive divisor of $\sigma$. In other words, the Eisenman intrinsic $k$-measure does not change under deleting of this exclusive divisor. Our attempts to show that this additional condition is necessary failed and, in fact, we found out that this behavior of Eisenman measures under blowing-ups remains the same in some other cases which will be discussed in section 6 of this paper. This enables us to answer positively to the following question of Zaidenberg: whether $E_{k}^{\tilde{M}-\tilde{A}}$ coincides with the restriction of $E_{k}^{\tilde{M}}$ to $\tilde{M}-\tilde{A}$ when $A$ is a retract of $M$.

Recall that $M$ is called (meromorphically) measure hyperbolic if its KobayashiEisenman (resp. Yau) pseudovolume is volume everywhere on $M$ except for, possibly, a subset of Hausdorff $2 m$-measure zero. In [Ka95] we present an example of a complex manifold which is measure hyperbolic but not meromorphically measure hyperbolic. This gives a negative answer to Kobayashi's question. It turns out that

Received by the editors June 19, 1995.

1991 Mathematics Subject Classification. Primary 32H20, 32H15.

(C)1996 American Mathematical Society 
the same example produces the negative answers to other questions which were, unfortunately, unknown to the author while he was preparing the earlier paper.

The first question (Demailly) is whether the fact that $M$ is measure hyperbolic implies the existence of a Hermitian metric on $M$ with a negatively bounded Ricci curvature. We discuss it in section 3.

In [G89] Gromov introduced the notions of a $\mathbf{C}$-connected manifold, a densely sub-Euclidean manifold, and a Runge manifold. A complex manifold is called $\mathbf{C}$ connected if any two points of this manifold lie in a holomorphic image of $\mathbf{C}$ in this manifold. A complex manifold is densely sub-Euclidean if it contains a dense image of a Euclidean space under a holomorphic mapping. A complex manifold of dimension $m$ is Runge if every holomorphic mapping from a ball of dimension $m$ into this manifold can be approximated on compacts by holomorphic mappings from $\mathbf{C}^{m}$. The second question (Gromov [G89, p. 881]) which will be discussed in sections 4 and 5 asks whether a $\mathbf{C}$-connected manifold must be densely subEuclidean or Runge.

\section{Preliminaries}

In this section we remind the formulations of the results from [Ka95] more accurately. We suppose that the definitions of the Eisenman and Yau intrinsic measures are known (e.g., see [E70], [Y75], [Ko76], [GW85]). Let $M, \tilde{M}, A, \tilde{A}, \sigma, \ell$ have the same meaning as in the introduction.

Definition. We call the divisor $\tilde{A}$ " $k$-negligible" if the measure $E_{k}^{\tilde{M}-\tilde{A}}$ coincides with the restriction of $E_{k}^{\tilde{M}}$ to $\tilde{M}-\tilde{A}$.

Theorem ([Ka95]). The divisor $\tilde{A}$ is k-negligible if one of the following conditions holds:

(i) $\ell \geq[(k-1) / 2]+2$ where $[(k-1) / 2]$ is the integral part of $(k-1) / 2$;

(ii) the Chern class $c_{\ell-1}\left(\mathcal{N}_{A}\right)$ of the normal bundle $\mathcal{N}_{A}$ of $A$ in $M$ is trivial.

In order to describe the counterexample to the question of Kobayashi we need one more fact, besides this theorem.

Lemma ([Ka95]). There exists a discrete set $T$ in $\mathbf{C}^{m}(m \geq 2)$ such that the manifold $L^{\prime}=\mathbf{C}^{m}-T$ is measure hyperbolic. Moreover, its Kobayashi-Eisenman pseudovolume is volume at each point of $L^{\prime}$.

After blowing $\mathbf{C}^{m}$ up at each point of $T$ one obtain a new manifold $\tilde{L}$ whose Yau pseudovolume is identically zero (since $\tilde{L}$ is bimeromorphically equivalent to $\mathbf{C}^{m}$ via the natural projection $\pi: \tilde{L} \rightarrow \mathbf{C}^{m}$ ). On the other hand, by the above Theorem and Lemma, the Kobayashi-Eisenman pseudovolume of $\tilde{L}$ is volume on $\tilde{L}-\tilde{T}$ where $\tilde{T}$ is the preimage of $T$ in $\tilde{L}$. Hence $\tilde{L}$ is measure hyperbolic and, therefore, it provides the negative answer to Kobayashi's question.

3.

We suppose that the definitions of the Ricci curvature of a Hermitian metric and a negatively bounded Ricci curvature are known (e.g., see [Ko76], [GW85]).

In order to answer the question of Demailly we need to apply the following remarkable fact [Ko76, Theorem 6.7]: 
Every complex manifold which admits a Hermitian volume whose Ricci curvature is negatively bounded must be meromorphically measure hyperbolic.

Now we see that $\tilde{L}$ cannot possess a Hermitian metric with negatively bounded Ricci curvature in spite of the fact that it is measure hyperbolic.

4.

Proposition. Suppose that the Kobayashi-Eisenman pseudovolume of a complex manifold $M$ is volume at least at one point $o$. Then $M$ is not Runge. If the Kobayashi-Eisenman pseudovolume is a volume on an open subset of $M$, then $M$ is not densely sub-Euclidean.

Proof. Consider a holomorphic mapping of a ball (which has the same dimension $m$ as $M$ ) into a coordinate neighborhood of $o$ such that $o$ is the image of the origin and the Jacobian of this mapping at the origin is nonzero (we fix some local coordinate system in the neighborhood of $o$ in order to determine the Jacobian). Assume that $M$ is Runge. Then there exists a holomorphic mapping from $\mathbf{C}^{m}$ to $M$ so that $o$ is the image of the origin and the Jacobian of this mapping at the origin is nonzero. Since the Kobayashi-Eisenman pseudovolume of $\mathbf{C}^{m}$ is identically zero, we see that the Kobayashi-Eisenman pseudovolume of $M$ must be zero at $o$, by the decreasing property of Eisenman measures under holomorphic mappings (e.g., see [GW85, Lemma 2.16]) (it follows also from the definition of Eisenman measures). This contradiction shows that our assumption that $M$ has the Runge property is wrong. Similarly, if $M$ contains a dense image of a Euclidean space under a holomorphic mapping, then the Kobayashi-Eisenman pseudovolume is identically zero on this image which proves the second statement.

Thus the manifold $L^{\prime}$ introduced in section 2 is not Runge since it is measure hyperbolic. It is easy to see that the Kobayashi-Royden pseudometric of $L^{\prime}$ is zero (e.g., see [KZ], [CHO76]). This shows already that the triviality of the KobayashiRoyden pseudometric is not the weakest ellipticity condition (see [G89, p. 881]). One can also see that a stronger fact holds for $L^{\prime}$.

Lemma. The manifold $L^{\prime}$ is $\mathbf{C}$-connected.

Proof. We consider the case of $m=2$ only. Then $L^{\prime} \subset \mathbf{C}^{2}$. Suppose that $(x, y)$ is a coordinate system in $\mathbf{C}^{2}$, and $w_{k}=\left(x_{k}, y_{k}\right)(k=1,2)$ are points in $L^{\prime}$. Without loss of generality we may suppose that $x_{1} \neq x_{2}$. Consider a polynomial $p \in \mathbf{C}[x]$ of degree 2 such that $p\left(x_{k}\right)=y_{k}(k=1,2)$ and consider the curve $\Gamma_{p}=\left\{(x, y) \in \mathbf{C}^{2} \mid\right.$ $y=p(x)\}$ which is isomorphic to $\mathbf{C}$. The set of all polynomials $p$ with the above property forms an algebraic curve $C$. Let $w \in T$. Then $w \in \Gamma_{p}$ only for at most one point $p \in C$. Hence for every $p$ which belongs to the complement of a countable subset in $C$ the curve $\Gamma_{p}$ does not meet $T$ while it contains $w_{1}$ and $w_{2}$.

Thus $L^{\prime}$ produces the negative answer to the question of Gromov.

5.

The manifold $L^{\prime}$ is not Stein. One may ask whether in the case of a Stein manifold the $\mathbf{C}$-connectedness implies that the manifold is densely sub-Euclidean or Runge. We show below that it is not so.

Consider again $\tilde{L}, T, m$, and $\pi$ from section 2. Suppose that $m=2$ and fix a coordinate system $(x, y)$ on $\mathbf{C}^{2}$. Let $T=\left\{w_{k}^{0}=\left(x_{k}^{0}, y_{k}^{0}\right) \mid k=1,2, \ldots\right\}$. Each 
curve $\pi^{-1}\left(w_{k}^{0}\right)$ in $\tilde{L}$ is isomorphic to $\mathbf{C P}^{1}$ which has the following homogeneous coordinate system:

$$
\left(\tilde{x}_{k}: \tilde{y}_{k}\right)=\left(\left(x-x_{k}^{0}\right):\left(y-y_{k}^{0}\right)\right) .
$$

Delete in every curve $\pi^{-1}\left(w_{k}^{0}\right)$ the point $(0: 1)$. We obtain a new manifold $\tilde{L}^{*}$ which is measure hyperbolic since $\tilde{L}$ is measure hyperbolic and $\tilde{L}^{*}$ is a dense submanifold of $\tilde{L}$. Thus $\tilde{L}^{*}$ is not Runge, by Proposition 4 .

Lemma. The manifold $\tilde{L}^{*}$ is Stein and $\mathbf{C}$-connected.

Proof. We check first that $\tilde{L}^{*}$ is $\mathbf{C}$-connected. Let $\tilde{w}_{k}(k=1,2)$ be points in $\tilde{L}^{*}$. If none of this points belongs to a curve $\pi^{-1}(w)$ for some $w \in T$, then these points lie in a holomorphic image of $\mathbf{C}$, by Lemma 4 . The same is, obviously, true when they belong to the same curve $\pi^{-1}(w)$. Thus we need to assume that either $\pi\left(\tilde{w}_{1}\right)$, or $\pi\left(\tilde{w}_{2}\right)$, or both of them are in $T$, and $\pi\left(\tilde{w}_{1}\right) \neq \pi\left(\tilde{w}_{2}\right)$. Consider, for instance, the case when $w^{k}:=\pi\left(\tilde{w}_{k}\right)$ is in $T$ for both $k=1,2$. Let $\left(\tilde{x}_{k}^{0}: \tilde{y}_{k}^{0}\right)$ be the homogeneous coordinates of $\tilde{w}_{k}$ in $\pi^{-1}\left(w^{k}\right)$ and let $w^{k}=\left(x_{k}, y_{k}\right)$. Consider a polynomial $p \in \mathbf{C}[x]$ of degree 4 such that $p\left(x_{k}\right)=y_{k}$ and $p^{\prime}\left(x_{k}\right)=\tilde{y}_{k}^{0} / \tilde{x}_{k}^{0}$ for $k=1,2$. Consider also the curve $\Gamma_{p}=\left\{(x, y) \in \mathbf{C}^{2} \mid y=p(x)\right\}$ which is isomorphic to $\mathbf{C}$. The set of all polynomials $p$ with the above property forms an algebraic curve $C$. Let $w \in T-\left\{w^{1}, w^{2}\right\}$. Then $w \in \Gamma_{p}$ only for at most one point $p \in C$. Hence for every $p$ which belongs to the complement of a countable subset in $C$ the curve $\Gamma_{p}$ does not meet $T-\left\{w^{1}, w^{2}\right\}$ while it contains $w^{1}$ and $w^{2}$. One can see that the proper transform of $\Gamma_{p}$ in $\tilde{L}$ is contained, actually, in $\tilde{L}^{*}$ and this proper transform contains the points $\tilde{w}_{1}$ and $\tilde{w}_{2}$. The case when only one of the points $\pi\left(\tilde{w}_{k}\right)(k=1,2)$ belongs to $T$ can be treated in the same manner. Thus $\tilde{L}^{*}$ is C-connected.

We suppose further that $\left|w_{n+1}^{0}\right| \geq\left|w_{n}^{0}\right|$ for every $n=1,2, \ldots$ Consider the blowing-up $\pi^{n}: L^{n} \rightarrow \mathbf{C}^{2}$ of $\mathbf{C}^{2}$ at the points $\left\{w_{k}^{0} \in T \mid k=1,2, \ldots, n\right\}$. Remove the point $(0: 1)$ from each curve $\left(\pi^{n}\right)^{-1}\left(w_{k}^{0}\right)$. We obtain a new manifold $L_{n}$. Denote by $\pi_{n}$ the restriction of $\pi^{n}$ to $L_{n}$. Then there is a natural projection $\tilde{\pi}_{n}: \tilde{L}^{*} \rightarrow L_{n}$ such that $\pi_{n} \circ \tilde{\pi}_{n}$ coincides with the restriction of $\pi$ to $\tilde{L}^{*}$. Let $B$ be an open ball in $\mathbf{C}^{2}$ with center at the origin and with radius $\left|w_{n}^{0}\right|$. Put $B_{n}=\pi_{n}^{-1}(B)$ and $\tilde{B}_{n}=\tilde{\pi}_{n}^{-1}\left(B_{n}\right)$. Note that the restriction of $\tilde{\pi}_{n}$ to $\tilde{B}_{n}$ is a biholomorphic mapping between $\tilde{B}_{n}$ and $B_{n}$. The manifold $L_{n}$ is Stein since it is isomorphic to the affine algebraic variety

$$
\left\{\left(x, y, z_{k}, \ldots, z_{n}\right) \in \mathbf{C}^{n+2} \mid y-y_{k}^{0}=z_{k}\left(x-x_{k}^{0}\right), k=1,2, \ldots, n\right\} .
$$

Thus every two points in $B_{n}$ may be distinguished by a holomorphic function $f$ on $L_{n}$. Hence every two points in $\tilde{B}_{n}$ may be distinguished by the holomorphic function $f \circ \tilde{\pi}_{n}$, which is the first condition needed for $\tilde{L}^{*}$ to be Stein.

Let $\tilde{K}$ be a compact in $\tilde{L}^{*}$ so that $K:=\pi(\tilde{K}) \subset B$. Put $K_{n}=\tilde{\pi}_{n}(\tilde{K})$. Note that the holomorphic hull of $K$ in $\mathbf{C}^{2}$ is contained in $B$. Hence the holomorphic hull $\hat{K}_{n}$ of $K_{n}$ in $L_{n}$ is contained in $B_{n}$ and the holomorphic hull of $\tilde{K}$ is contained in $\tilde{B}_{n}$. Since $L_{n}$ is Stein, $\hat{K}_{n}$ is compact. Since $\tilde{B}_{n}$ is biholomorphic to $B_{n}$ via $\tilde{\pi}_{n}$, we see that the holomorphic hull of $\tilde{K}$, which is contained in $\tilde{\pi}_{n}^{-1}\left(\hat{K}_{n}\right)$, is also compact. Hence $\tilde{L}^{*}$ is holomorphically convex and, therefore, Stein.

Therefore, one can see that $\tilde{L}^{*}$ is a $\mathbf{C}$-connected Stein manifold which is not densely sub-Euclidean or Runge. 
6.

The aim of this section is to strengthen Theorem 2. We shall use the following notation: $\quad M, \tilde{M}, A, \tilde{A}, \sigma, \ell$ have the same meaning as in the introduction, $S$ is a Stein manifold, $\tilde{F}: S \rightarrow \tilde{M}$ is a holomorphic mapping whose image is not contained completely in $\tilde{A}$, and $F=\sigma \circ \tilde{F}$. We consider the graphs $\Gamma \subset N:=S \times M$ and $\tilde{\Gamma} \subset \tilde{N}:=S \times \tilde{M}$ of the mappings $F$ and $\tilde{F}$ respectively. Denote the mapping $(i d, \sigma): \tilde{N} \rightarrow N$ by $\tau$. Let $\rho: \Gamma \rightarrow M$ and $\tilde{\rho}: \tilde{\Gamma} \rightarrow \tilde{M}$ be the natural projections. Put $E=\rho^{-1}(A)$ and $\tilde{E}=\tilde{\rho}^{-1}(\tilde{A})$. Consider the following holomorphic vector bundles on $E$ : $W^{\prime}$ is the restriction of the tangent bundle $T N$ of $N$ to $E, W_{0}$ is the bundle $\tau_{*}\left(\left.T \tilde{N}\right|_{\tilde{E}}\right)$ over $E$ where $T \tilde{N}$ is the tangent bundle of $\tilde{N}\left(W_{0}\right.$ is a holomorphic bundle, by [Ka95, Lemma 3.2]), and $W^{\prime \prime}$ is the quotient bundle $W^{\prime} / W_{0}$ whose rank is $\ell-1$ [Ka95]. By the Oka-Weil-Grauert principle (e.g., see [L86]), if $W^{\prime \prime}$ has a continuous nowhere zero section, it has a similar holomorphic section. Since $W^{\prime \prime}$ has this continuous section only if the Chern class $c_{\ell-1}\left(W^{\prime \prime}\right)=0$ (e.g., see [Hi66]), we can reformulate Lemma 3.4 from [Ka95] as follows.

Proposition. Let $R$ be a relatively compact submanifold of $S$ and let $q$ be a point in the closure $\bar{R}$ of $R$ for which $F(q) \notin A$. If $c_{\ell-1}\left(W^{\prime \prime}\right)=0$, then there exists a family of holomorphic mappings $F_{t}$ from a neighborhood $U \subset S$ of $\bar{R}$ into $M$ depending holomorphically on parameter $t$ from the unit disc and such that

(1) the restriction of $F$ to $U$ coincides with $F_{0}$;

(2) for every $t$ we have $F_{t}(q)=F(q)$ and $\left.F_{t *}\right|_{T_{q} S}=\left.F_{*}\right|_{T_{q} S}$, where $T_{q} S$ is the tangent space of $S$ at $q$;

(3) $F_{t}(\bar{R})$ does not meet $A$ for any $t \neq 0$.

Lemma. Suppose that $H^{r}(S, \mathbf{Z})=0$ for $r=2$ and $r=2 \ell-2$. Let $i: A \rightarrow M$ and $\tilde{i}: \tilde{A} \rightarrow \tilde{M}$ be natural embeddings. Suppose that either

(1) the mapping $i^{*}: H^{2 \ell-2}(M, \mathbf{Z}) \rightarrow H^{2 \ell-2}(A, \mathbf{Z})$ is surjective, or

(2) the mapping $\tilde{i}^{*}: H^{2 \ell-2}(\tilde{M}, \mathbf{Z}) \rightarrow H^{2 \ell-2}(\tilde{A}, \mathbf{Z})$ is surjective.

Then $c_{\ell-1}\left(W^{\prime \prime}\right)=0$ and, therefore, there exists a family of holomorphic mappings $F_{t}$ described in the previous Proposition.

Proof. Let $\rho_{0}$ be the restriction of $\rho$ to $E$. It was shown in the the proof of Proposition 3.5 from [Ka95] that the direct sum of $W^{\prime \prime}$ and a trivial line bundle may be viewed as the pullback $W=\rho_{0}^{*}\left(\mathcal{N}_{A}\right)$ of the normal bundle $\mathcal{N}_{A}$ of $A$ in $M$. (Condition that $H^{2}(S, \mathbf{Z})=0$ is needed for this claim.) By the Whitney formula [Hi66] the total Chern classes of $W$ and $W^{\prime \prime}$ coincide. In particular, $c_{\ell-1}\left(W^{\prime \prime}\right) \in \rho_{0}^{*}\left(H^{2 \ell-2}(A, \mathbf{Z})\right)$.

Consider the commutative diagram

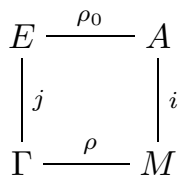

where $j: E \rightarrow \Gamma$ is the natural embedding. It generates the commutative diagram

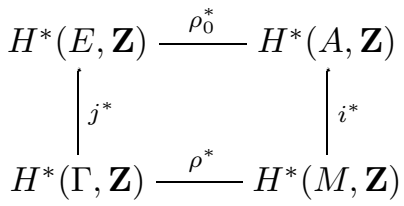


From this diagram we see that if $i^{*}$ is an epimorphism, then $c_{\ell-1}\left(W^{\prime \prime}\right)$ belongs not only to $\rho_{0}^{*}\left(H^{2 \ell-2}(A, \mathbf{Z})\right)$ but also to $j^{*}\left(H^{2 \ell-2}(\Gamma, \mathbf{Z})\right)$. Since $\Gamma$ is biholomorphic to $S$, we have $H^{2 \ell-2}(\Gamma, \mathbf{Z})=0$, which implies (1).

Let $\tilde{\rho}_{0}$ be the restriction of $\tilde{\rho}$ to $\tilde{E}$. Then $\rho_{0}$ is the composition of $\tilde{\rho}_{0}$ and the natural projection $\tilde{A} \rightarrow A$. Hence $c_{\ell-1}\left(W^{\prime \prime}\right)$ belongs to $\tilde{\rho}_{0}^{*}\left(H^{2 \ell-2}(\tilde{A}, \mathbf{Z})\right)$. If $\tilde{i}^{*}$ is an epimorphism, then in the same way as above one can show that $c_{\ell-1}\left(W^{\prime \prime}\right) \in$ $\tilde{j}^{*}\left(H^{2 \ell-2}(\tilde{\Gamma}, \mathbf{Z})\right)$, where $\tilde{j}: \tilde{E} \rightarrow \tilde{\Gamma}$ is the natural embedding. Since $\tilde{\Gamma}$ is biholomorphic to $S$, we have $H^{2 \ell-2}(\tilde{\Gamma}, \mathbf{Z})=0$ which implies (2).

Applying this Lemma to the case when $S$ is a $k$-dimensional ball (see the proof of Theorem A in [Ka95]) we can obtain the following

Theorem. The divisor $\tilde{A}$ is k-negligible if one of the following conditions holds:

(iii) the mapping $i^{*}: H^{2 \ell-2}(M, \mathbf{Z}) \rightarrow H^{2 \ell-2}(A, \mathbf{Z})$ is surjective, or

(iv) the mapping $\tilde{i}^{*}: H^{2 \ell-2}(\tilde{M}, \mathbf{Z}) \rightarrow H^{2 \ell-2}(\tilde{A}, \mathbf{Z})$ is surjective.

\section{Concluding Remarks}

1. The positive answer to Zaidenberg's question mentioned in the introduction follows, of course, from Theorem 6 (iii). (This question was our motivation to write down Theorem 6.)

2. We do not know if conditions (i)-(iv) in Theorems 2 and 6 can be removed or chosen weaker.

3. Let $\delta: \hat{M} \rightarrow M$ be the composition of blowing-ups $\sigma_{r}: M_{r} \rightarrow M_{r-1}$ where $r=1, \ldots, n, M=M_{0}$, and $M_{n}=\hat{M}$. Let $A_{r-1}$ be the centrum of $\sigma_{r}$, let $\delta_{r}=\sigma_{r} \circ \cdots \circ \sigma_{1}$, let $D=A_{0} \cup \bigcup_{r=1}^{n-1} \delta_{r}\left(A_{r}\right)$, and let $\hat{D}=\delta^{-1}(D)$. Suppose that for every $r=0, \ldots, n-1$ the divisor $A_{r}$ in $M_{r}$ satisfies one of conditions (i)-(iv) from Theorems 2 and 6 for some fixed $k$. Then several applications of Proposition 6 show that $\hat{D}$ is $k$-negligible.

4. In particular, if $\delta: \hat{M} \rightarrow M$ is a birational morphism of algebraic surfaces, then $\hat{D}$ is $k$-negligible for every $k$. (This follows from Theorem 2 (i) since the centrum of a blowing-up is always a point for surfaces.)

5 . We do not know if a measure hyperbolic compact (or algebraic) manifold must be meromorphically measure hyperbolic. More generally, what condition must we impose on the manifold in order to have a positive answer to Kobayashi's question? In the Stein situation the answer is positive, since the Eisenman and Yau measures coincide.

6. Similarly, we do not know whether a C-connected compact (or algebraic) manifold is densely sub-Euclidean or Runge.

\section{REFERENCES}

[CHO76] L. Campbell, A. Howard, T. Ochiai, Moving discs off analytic subsets, Proc. AMS, 60(1976), 106-107. MR 54:13143

[E70] D. A. Eisenman, Intrinsic measures on complex manifolds and holomorphic mappings, Mem. AMS, No. 96, AMS, Providence, R. I., 1970. MR 41:3807

[GW85] I. Graham, H. Wu, Some remarks on the intrinsic measures of Eisenman, Trans. AMS, 288 (1985), 625-660. MR 86e:32031

[G89] M. Gromov, Oka's principle for holomorphic sections of elliptic bundles, J. AMS, 2 (1989), 851-897. MR 90g:32017

[Hi66] F. Hirzebruch, Topological methods in algebraic geometry, Springer-Verlag, New York, 1966. MR 34:2573 
[Ka95] Sh. Kaliman, Some facts about Eisenman intrinsic measures, Compl. Variables, 27 (1995), 163-173. MR 96b:32032

[KZ] Sh. Kaliman, M. Zaidenberg, A transversality theorem for holomorphic mappings and stability of Eisenman-Kobayashi measures, Trans. AMS (to appear). CMP 95:09

[Ko76] S. Kobayashi, Intrinsic distances, measures and geometric function theory, Bull. AMS, 82 (1976), 357-416. MR 54:3032

[L86] J. Leiterer, Holomorphic vector bundles and the Oka-Grauert principle, Current problems in mathematics. Fundamental directions, vol. 10, Itogi Nauki i Tekhniki, Akad. Nauk SSSR, Vsesoyuz. Inst. Nauchn. i Tekhn. Inform., Moscow, 1986, pp. 75-121. (Russian) MR 88k:32072

[Y75] S.-T. Yau, Intrinsic measures of compact complex manifolds, Math. Ann. 212 (1975), 317-329. MR 51:3503

Department of Mathematics and Computer Science, University of Miami, Coral GABLES, FLORIDA 33124

E-mail address: kaliman@paris.cs.miami.edu 